\title{
Congenital humeroradial synostosis
}

\author{
Zhen Jiang Liu • Qun Zhao
}

Received: 19 March 2010/Revised: 1 May 2010/Accepted: 14 May 2010 /Published online: 10 June 2010

(C) Springer-Verlag 2010

We present a $2 \frac{1}{2}$-year-old boy with congenital bilateral humeroradial synostoses. There was no family history of skeletal malformations. Radiographs showed bilateral humeroradial synostoses without ulnar ray hypoplasia (Fig. 1). There were no humeroulnar articulation synostoses. No specific syndromic features were noted. His bilateral elbows were fixed in flexion deformity. He had normal interphalangeal joints, and shoulder, hip, knee and ankle joints. There was no limb hypoplasia. The parents refused operative treatment.

Congenital bilateral humeroradial synostoses are rare [1]. The current classification divides the synostosis into Class I (fixed in extension with ulnar ray hypoplasia and sporadic) and Class II (fixed in flexion without hypoplasia and familial). Class II may be associated with multiple systemic anomalies [2]. Elbow synostosis often produces little functional disability [1]. Although soft tissue release and osteotomy may initially improve range of movement [1], re-ossification is the norm.

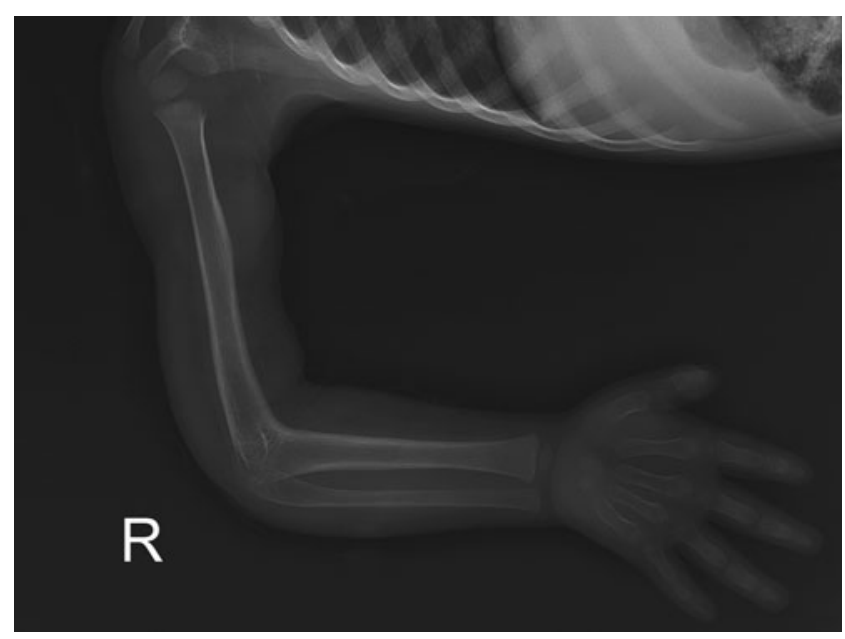

Fig. 1 Lateral radiograph, right elbow

\section{References}

1. McIntyre JD, Benson MK (2002) An aetiological classification for developmental synostoses at the elbow. J Pediatr Orthop B 11:313319

2. McIntyre JD, Brooks A, Benson MK (2003) Humeroradial synostosis and the multiple synostosis syndrome: case report. J Pediatr Orthop B 12:192-197 